\title{
Hall Effects on Unsteady Magneto Hydrodynamic Convection Flow of Nanofluids Past a Rotating Porous Plate
}

\author{
Pushpabaipavar, L. Harikrishna, M. Suryanarayana Reddy
}

\begin{abstract}
The effects of Hall current are considered for the convective rotational current free of nanofluid magnetohydrodynamics (copper and alumina) in a permeable medium with a vertical porous flat plate, semi-infinite rotation with stable state of the heat source and convection limit. The slip rate is expected to oscillate over time with a constant frequency so that the boundary layer solutions are of the equivalent oscillating type. The equations to regulate the flow are analytically solved by perturbation estimation. The effects of different parameters on the flow are investigated by means of diagrams and tables.
\end{abstract}

Keywords: Porous medium; Nanofluids; Convective flow; rotating frame; Heat transfer.

\section{NOMENCLATURE}

$u, v, w$ velocity components along $x, y$ andz-axis respectively.

$\beta_{n f} \quad$ coefficient of the thermal expansion.

$K_{n f} \quad$ Thermal conductivity.

$U_{r} \quad$ The uniform reference velocity.

$\varepsilon \quad$ The small constant quantity.

$\sigma \quad$ Electric conductivity.

$\rho_{n f} \quad$ Density.

$\mu_{n f} \quad$ viscosity.

$\left(\rho C_{p}\right)_{n f}$ heat capacitance.

$g$ acceleration due to gravity.

$k$ permeability of porous medium.

$T$ Temperature.

$Q \quad$ Temperature dependent volumetric rate of heat source.

$\alpha_{n f} \quad$ Thermal diffusivity.

$(\rho \beta)_{n f}$ The thermal expansion coefficient of the nanofluid $\phi \quad$ Solid volume fraction of the nanoparticles.

Revised Manuscript Received on December 30, 2019.

* Correspondence Author

Pushpabaipavar, Research Scholar, Department of Mathematics, JNTU Anantapur (Andhra Pradesh) India. E-mail: Pushpapavar66@gmail.com

L. Harikrishna*, Department of Mathematics (H\&S), Annamacharya Institute of Technology and Sciences, Rajampet (Andhra Pradesh) India. E-mail: lhkmaths@gmail.com

M. Suryanarayana Reddy, Assistant Professor, Department of Mathematics, JNTUA College of Engineering, Pulivendula (AndhraPradesh) India. E-mail: machireddysnr.maths@jntua.ac.in

(C) The Authors. Published by Blue Eyes Intelligence Engineering and Sciences Publication (BEIESP). This is an open access article under the CC BY-NC-ND license (http://creativecommons.org/licenses/by-nc-nd/4.0/) $w_{0}$ The normal velocity at the plate.

$v_{f} \quad$ Kinematic viscosity.

$R$ rotational parameter.

$M$ magnetic field parameter.

Pr Prandtl number.

$S \quad$ Suction $(\mathrm{S}>0)$ or injection $(\mathrm{S}<0)$ parameter.

$K \quad$ Permeability of the porous medium and

$Q_{H} \quad$ Heat source parameter

$B_{0} \quad$ Magnetic induction

$k$ Permeability of porous medium

$\Omega \quad$ Angular velocity

$\sigma \quad$ Electrical conductivity of the fluid

$\gamma \quad$ Convective parameter

$R e_{x} \quad$ Local Reynolds number

$\tau \quad$ Skin friction parameter

$\mathrm{Nu} \quad$ Nusselt number

$n \quad$ Frequency of oscillation

$t$ Time

Subscripts:

$f \quad$ Base fluid

nf Nano-fluid

$s \quad$ Nanosolid paricles

\section{INTRODUCTION}

There is very significant interest in science and technology for convective Nano fluid heat transfer. Ethylene glycol, Water, and engine oil are heating or cooling agents and play a decisive role in many industries ' thermal management with low thermal conductivity. We enhance thermal conductivity for extended surfaces, mini-channels and micro-channels. Concrete materials have higher thermal conductivities.

The word nanofluid has been presented first by Choi [3]. Nano-particles are a viaduct between enormity materials and nuclear or molecular sytheses. Some of nano-particles have utilized are $\mathrm{Al}, \mathrm{Cu}, \mathrm{Fe}$ and $\mathrm{Ti}$ or their oxides. Progressed nuclear system [1] has great application utilizing of nanofluids. Micro-channel miniaturization and cooling of the system, heat transfer system size decrease, improved heat transfer and negligible clogging are the advantages of nanofluids. Conge do et al. [5], Das, Kalidas [11] and Ghasemi discussed on regular convection heat transfer in nanofluids. The 2-dimensional regular convection flow of a nanofluid in a walled in area has been discuused by Khanafer et al. [4]. MHD Non-Newtonian fluid rotating streams have various uses in turbo equipment, geophysics, meteorology, and a few areas. Das, Kalidas[11], Bakr6] and Das[9] investigated micropolar fluid free convection flow in a pivoting stream.

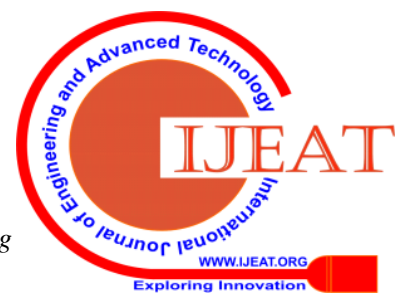


As of late, Hamad and Pop [7] researched free convective MHD pivoting flow in a nanofluid with steady heat source. Kalidas[11] have suggested the new nanofluid model. Makinde and Aziz [8], Das, Kalidas [11] and Yacob et al. [10] discussed the heat transfer hitches for the flow of the boundary layer with a special type of boundary condition. Veera Krishna et al. [12] investigated the effects of Hall current on magnetohydrodynamic currents through porous channels. Taking into account the aforementioned facts, the effects of Hall on the central flow of nanofluid unstable magnetohydrodynamic free convection (copper and alumina) in a permeable medium are calculated.

\section{FORMULATION AND SOLUTION OF THE PROBLEM}

We consider the effects of Hall on the unstable free convection flow of nanofluids at room temperature (copper and alumina) on a permeable plate in vertical semi-infinite movement embedded in a homogeneous porous medium under the influence of thermal buoyancy with a stable heat source and A special kind of boundary conditions. We assume that the nanoparticles had a uniform shape and size. There is also thermal equilibrium in both nanoparticles and liquid phase.

Image. Fig. 1 Images the physical problem model. It is assumed that the flow is in the $\mathrm{x}$-direction obtained in the ascendant direction along the sheet, and it is usual for z-axis. The whole device is rotated at an angular velocity around $\mathrm{z}$-axis. It is assumed that an invariable peripheral magnetic field $\mathrm{B} 0$ acts along the z-axis. Voltage also does not apply ( $\mathrm{E}$ $=0$ ). The induced magnetic field is small in contrast to the outer magnetic field. Therefore, the Reynolds magnetic number is low for the oscillating plate. Because of the assumption of a semi-infinite platelayer, each of the variables is as if they were elements of $\mathrm{z}$ and time.

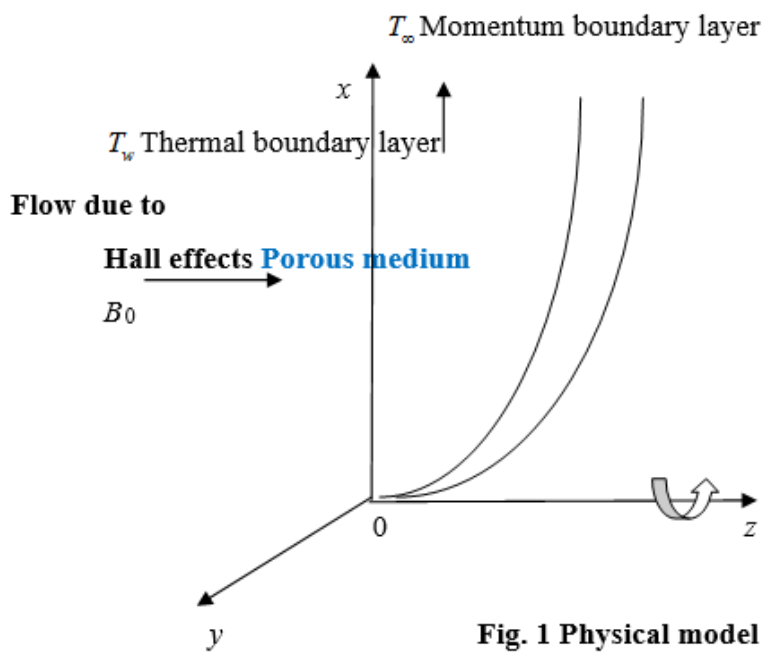

The basic conditions that depict the physical circumstances are given under the approximations of the limit layer $\frac{\partial w}{\partial z}=0$

(1)

$\frac{\partial u}{\partial t}+w \frac{\partial u}{\partial z}-2 \Omega v=\frac{\mu_{n f}}{\rho_{n f}} \frac{\partial^{2} u}{\partial z^{2}}-\frac{\mu_{n f} u}{\rho_{n f} k}+B_{0} J_{y}+g \beta_{n f}\left(T-T_{\infty}\right)$

(2) $\frac{\partial v}{\partial t}+w \frac{\partial v}{\partial z}+2 \Omega u=\frac{\mu_{n f}}{\rho_{n f}} \frac{\partial^{2} v}{\partial z^{2}}-\frac{\mu_{n f} v}{\rho_{n f} k}-B_{0} J$

(3)

$\frac{\partial T}{\partial t}+w \frac{\partial T}{\partial z}=\alpha_{n f} \frac{\partial^{2} T}{\partial z^{2}}-\frac{Q}{\left(\rho C_{p}\right)_{n f}}\left(T-T_{\infty}\right)$

The conditions are determined by $u=v=0, \quad T=T_{\infty}$ for $t \leq 0$

(5) $\left.\begin{array}{l}u=U_{r}\left[1+\frac{\varepsilon}{2}\{\exp (\text { int })+\exp (-\mathrm{int})\}\right], v=0,-K_{n f} \frac{\partial T}{\partial z}=h_{f}\left(T_{w}-T_{\infty}\right) \text { at } z=0 \\ u \rightarrow 0, \quad v \rightarrow 0, \quad T \rightarrow T_{\infty} \quad \text { as } \quad z \rightarrow \infty\end{array}\right\}$ for $t>0$

(6)

At the point when the value of the magnetic field is enormous, the description of the law of Ohm is modified to integrate the condition of the Hall so that

$\boldsymbol{J}+\frac{\omega_{e} \tau_{e}}{B_{0}}(\boldsymbol{J} \times \boldsymbol{B})=\sigma\left[\boldsymbol{E}+\boldsymbol{V} \times \boldsymbol{B}+\frac{1}{e \eta_{e}} \nabla P_{e}\right]$

Ionic and thermoelectric impacts are excluded. We press $\omega_{e} \tau_{e} \sim 0(1)$ and $\omega_{i} \tau_{i}<<1$, where $\omega_{i}$ and $\tau_{i}$ are respectively the cyclotron frequency and the collision time for the ions. Likewise, we expect that $\mathrm{E}=0$ under suspicions will decrease to

$J_{x}+m J_{y}=\sigma B_{0} v$

(8)

$J_{y}-m J_{x}=-\sigma B_{0} u$

Solving equations (8) and (8.a) we get,

$J_{x}=\frac{\sigma B_{0}}{1+m^{2}}(v+m u)$

$J_{y}=\frac{\sigma B_{0}}{1+m^{2}}(m v-u)$

We are replacing the equations (9) and (10) respectively in (3) and (2)

$\frac{\partial u}{\partial t}+w \frac{\partial u}{\partial z}-2 \Omega v=\frac{\mu_{n f}}{\rho_{n f}} \frac{\partial^{2} u}{\partial z^{2}}-\frac{\mu_{n f} u}{\rho_{n f} k}+\frac{\sigma B_{0}^{2}}{1+m^{2}}(m v-u)+g \beta_{n f}\left(T-T_{\infty}\right)$

$\frac{\partial v}{\partial t}+w \frac{\partial v}{\partial z}+2 \Omega u=\frac{\mu_{n f}}{\rho_{n f}} \frac{\partial^{2} v}{\partial z^{2}}-\frac{\mu_{n f} v}{\rho_{n f} k}-\frac{\sigma B_{0}^{2}}{1+m^{2}}(v+m u)$

The velocity of the oscillatory plate assumed in equation (6) The effective nanofluid density is determined by

$\rho_{n f}=(1-\phi) \rho_{f}+\phi \rho_{s}$

Thermal diffusivity of the nanofluid is

$\alpha_{n f}=\frac{K_{n f}}{\left(\rho C_{\mathrm{p}}\right)_{n f}}$

Where, the nanofluid's heat capacitance $\mathrm{Cp}$

$\left(\rho C_{p}\right)_{n f}=(1-\phi)\left(\rho C_{p}\right)_{f}+\phi\left(\rho C_{p}\right)_{s}$

The thermal conductivity of the naofluid $k_{n f}$ for spherical nanoparticles is as Maxwell

$$
\frac{k_{n f}}{k_{f}}=\frac{\left(k_{s}+2 k_{f}\right)-2 \phi\left(k_{f}-k_{s}\right)}{\left(k_{s}+2 k_{f}\right)+2 \phi\left(k_{f}-k_{s}\right)}
$$


The coefficient of thermal expansion of nanofluids is

$(\rho \beta)_{n f}=(1-\phi)(\rho \beta)_{f}+\phi(\rho \beta)_{s}$

Finally the effective dynamic viscosity of the given nanofluid $\mu_{n f}=\frac{\mu_{f}}{(1-\phi)^{2.5}}$

The thermo-physical properties of the nanofluids are given in Table 1.

$w=-w_{0}$

Where, $w_{0}$ is the normal velocity

Let us add variables that are dimensionless:

$u^{\prime}=\frac{u}{U_{r}}, v^{\prime}=\frac{v}{U_{r}}, z^{\prime}=\frac{z U_{r}}{v_{f}}, t^{\prime}=\frac{t U_{r}^{2}}{v_{f}}, n^{\prime}=\frac{n v_{f}}{U_{r}^{2}}$,

$$
\theta=\frac{\left(T-T_{\infty}\right)}{\left(T_{w}-T_{\infty}\right)} \quad R=\frac{2 \Omega v_{f}}{U_{r}^{2}}, \quad M=\frac{B_{0}}{U_{r}} \sqrt{\frac{\sigma v_{f}}{\rho_{f}}},
$$

$\operatorname{Pr}=\frac{v_{f}}{\alpha_{f}}, S=\frac{w_{0}}{U_{r}}, K=\frac{k U_{r}^{2}}{v_{f}^{2}} Q_{H}=\frac{Q v_{r}^{2}}{U_{r}^{2} k_{f}}$

Using above non-dimensional variables in equations (2)-(4) we obtain the following dimensionless equations

$$
\begin{aligned}
& {\left[1-\varphi+\varphi\left(\frac{\rho_{s}}{\rho_{f}}\right)\right]\left(\frac{\partial u}{\partial t}-S \frac{\partial u}{\partial z}-R v\right)=} \frac{1}{(1-\varphi)^{25}} \frac{\partial^{2} u}{\partial z^{2}}+\left[1-\varphi+\varphi\left(\frac{(\rho \beta)_{s}}{(\rho \beta)_{f}}\right)\right] \theta \\
&+\frac{\sigma B_{0}^{2}}{1+m^{2}}(m v-u)-\frac{u}{K} \\
& {\left[1-\varphi+\varphi\left(\frac{\rho_{s}}{\rho_{f}}\right)\right]\left(\frac{\partial v}{\partial t}-S \frac{\partial v}{\partial z}+R u\right)=} \frac{1}{(1-\varphi)^{2.5}} \frac{\partial^{2} v}{\partial z^{2}}-\frac{\sigma B_{0}^{2}}{1+m^{2}}(v+m u)-\frac{v}{K} \\
& {\left[1-\varphi+\varphi\left(\frac{\left(\rho C_{p}\right)_{s}}{\left(\rho C_{p}\right)_{f}}\right)\right]\left(\frac{\partial \theta}{\partial t}-S \frac{\partial \theta}{\partial z}\right)=\frac{1}{\operatorname{Pr}}\left(\frac{k_{n f}}{k_{f}} \frac{\partial^{2} \theta}{\partial z^{2}}-Q_{H} \theta\right) }
\end{aligned}
$$

The attribute of velocity is $\mathrm{Ur}$

$U_{r}=\left[g \beta_{f}\left(T_{w}-T_{\infty}\right) v_{f}\right]^{\frac{1}{3}}$

The borders are

$\mathrm{u}=\mathrm{v}=0, \theta=0, \quad$ for $\mathrm{t} \leq 0$

$\left.\begin{array}{l}u=1+\frac{\varepsilon}{2}\{\exp (\text { int })+\exp (-\mathrm{int})\}, \quad v=0, \quad \theta^{\prime}(0)=-\gamma(1-\theta(0)) \text { at } z=0 \\ u \rightarrow 0, \quad v \rightarrow 0, \quad \theta \rightarrow 0 \quad \text { as } \quad z \rightarrow \infty\end{array}\right\}$ for $t>0$

Here $\gamma=\frac{h_{f} v_{f}}{K_{f} U_{r}}$ is the connective parameter.

We now combining the equations (21) and (22) by substituting the fluid velocity in the form as (let $q=u+i v$ ). We're having it

$\left[1-\varphi+\varphi\left(\frac{\rho_{s}}{\rho_{t}}\right)\right]\left(\frac{\partial q}{\partial t}-S \frac{\partial q}{\partial z}+2 i R q\right)=\frac{1}{(1-\varphi)^{25}} \frac{\partial^{2} q}{\partial z^{2}}+\left[\left(1-\varphi+\varphi\left(\frac{(\rho \beta)_{s}}{(\rho \beta)_{t}}\right)\right) \theta-\left(\frac{M^{2}}{1-i m}+\frac{1}{K}\right) q\right]$

The conditions for the boundaries (24) and (25) are as follows:

$V=0, \theta=0$ for $\mathrm{t} \leq 0$

(27)

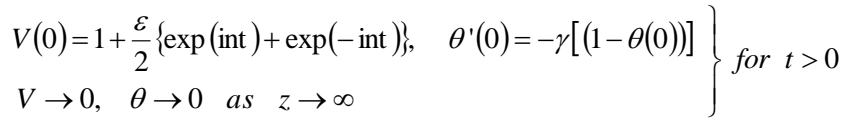

In order to locate the logical arrangements for the

arrangement of partial differential conditions (23), (26) in the plate region under the limit conditions (27), (28), we transmit, as well as (Das [11]).

$q(z, t)=q_{0}+\frac{\varepsilon}{2}\left[\exp (\right.$ int $) q_{1}(z)+\exp (-$ int $\left.) q_{2}(z)\right]$

$\theta(z, t)=\theta_{0}+\frac{\varepsilon}{2}\left[\exp (\right.$ int $) \theta_{1}(z)+\exp (-$ int $\left.) \theta_{2}(z)\right]$

For $\varepsilon(<<1)$.Recalling the previous equations (29) and (30) in equations (23) and (26) respectively. We obtain the following equations by matching non-harmonic and harmonic terms and neglecting the higher order conditions of $\varepsilon^{2}$. We get the following comparisons:

$$
\begin{gathered}
\frac{1}{(1-\varphi)^{2.5}} q_{0} "+S\left(1-\varphi+\varphi\left(\frac{\rho_{s}}{\rho_{f}}\right)\right) q_{0}{ }^{\prime}-\left[2 i R\left\{1-\varphi+\varphi\left(\frac{\rho_{s}}{\rho_{f}}\right)\right\}+\frac{M^{2}}{1-i m}+\frac{1}{K}\right] q_{0} \\
+\left[1-\varphi+\varphi\left(\frac{(\rho \beta)_{s}}{(\rho \beta)_{f}}\right)\right] \theta_{0}=0
\end{gathered}
$$

$$
\begin{gathered}
\frac{1}{(1-\varphi)^{25}} q_{1}{ }^{\prime \prime}+S\left(1-\varphi+\varphi\left(\frac{\rho_{s}}{\rho_{f}}\right)\right) q_{1}{ }^{\prime}-\left[i(2 R+n)\left\{1-\varphi+\varphi\left(\frac{\rho_{s}}{\rho_{f}}\right)\right\}+\frac{M^{2}}{1-i m}+\frac{1}{K}\right] q_{1} \\
+\left[1-\varphi+\varphi\left(\frac{(\rho \beta)_{s}}{(\rho \beta)_{f}}\right)\right] \theta_{1}=0 \\
\frac{1}{(1-\varphi)^{25}} q_{2}{ }^{\prime \prime}+S\left(1-\varphi+\varphi\left(\frac{\rho_{s}}{\rho_{t}}\right)\right) q_{2}{ }^{\prime}-\left[i(2 R-n)\left\{1-\varphi+\varphi\left(\frac{\rho_{s}}{\rho_{f}}\right)\right\}+\frac{M^{2}}{1-i m}+\frac{1}{K}\right] q_{2} \\
+\left[1-\varphi+\varphi\left(\frac{(\rho \beta)_{s}}{(\rho \beta)_{f}}\right)\right] \theta_{2}=0
\end{gathered}
$$

$\frac{k_{n f}}{k_{f}} \theta_{0}{ }^{\prime}+\operatorname{Pr} S\left[1-\phi+\phi\left(\frac{\left(\rho C_{p}\right)_{s}}{\left(\rho C_{p}\right)_{f}}\right)\right] \theta_{0}{ }^{\prime}-Q_{H} \theta_{0}=0$

$\frac{k_{n f}}{k_{f}} \theta_{1}{ }^{\prime}+\operatorname{Pr} S\left[1-\phi+\phi\left(\frac{\left(\rho C_{p}\right)_{s}}{\left(\rho C_{p}\right)_{f}}\right)\right] \theta_{1}{ }^{\prime}-\left[\right.$ in $\left.\operatorname{Pr}\left\{1-\phi+\phi\left(\frac{\left(\rho C_{p}\right)_{s}}{\left(\rho C_{p}\right)_{f}}\right)\right\}+Q_{H}\right] \theta_{1}=0$

$\frac{k_{n f}}{k_{f}} \theta_{2}{ }^{\prime \prime}+\operatorname{Pr} S\left[1-\phi+\phi\left(\frac{\left(\rho C_{p}\right)_{s}}{\left(\rho C_{p}\right)_{f}}\right)\right] \theta_{21}{ }^{\prime}-\left[\right.$ in $\left.\operatorname{Pr}\left\{1-\phi+\phi\left(\frac{\left(\rho C_{p}\right)_{s}}{\left(\rho C_{p}\right)_{f}}\right)\right\}-Q_{H}\right] \theta_{2}=0$

The boundary conditions are:

$q_{0}=q_{1}=q_{2}=1, \quad \theta_{0}{ }^{\prime}=-\gamma\left(1-\theta_{0}\right), \theta_{1}{ }^{\prime}=\gamma \theta_{1}, \quad \theta_{2}{ }^{\prime}=\gamma \theta_{2} \quad$ at $\mathrm{Z}=0$

$q_{0} \rightarrow 0, q_{1} \rightarrow 0, q_{2} \rightarrow 0, \theta_{0} \rightarrow 0, \theta_{1} \rightarrow 0, \theta_{2} \rightarrow 0$ as $z \rightarrow \infty$

Conditions for solving (25) - (30) under conditions (31), (32) are expressed as temperature and velocity 
$q=A_{1} \exp \left(-m_{1} z\right)+\left(1-A_{1}\right) \exp \left(-m_{2} z\right)+\left(\frac{\varepsilon}{2}\right)\left[\exp \left(-m_{3} z+\operatorname{int}\right)+\exp \left(-m_{4} z-\operatorname{int}\right)\right]$

$$
\theta=\frac{\gamma}{m_{1}+\gamma} \exp \left(-m_{1} z\right)
$$

We see the solution (39) and (40) approaching the constant s urface temperature solutions as $\gamma \rightarrow \infty$ an alternative. From the boundary conditions this can be seen (28), which gives $\theta(0)=1$ as $\gamma \rightarrow \infty$. It should also be noted that conditions (39) and (40) reduced to those of Hamad and Pop [7 ] when $m=0$, $K \rightarrow \infty$ and $\gamma \rightarrow \infty$.

The coefficient of skin friction $\mathrm{C}_{\mathrm{f}}$ and Nusselt number $\mathrm{N}_{\mathrm{u}}$ described as

$$
\begin{aligned}
C_{f} & =\frac{\left(T_{w}\right)_{z=0}}{\rho_{f} U_{t}^{2}}=\frac{1}{(1-\phi)^{2.5}} V^{\prime}(0) \\
& =-\frac{1}{(1-\phi)^{2.5}}\left[A_{1} m_{1}+\left(1-A_{1}\right) m_{2}+\left(\frac{\varepsilon}{2}\right)\left[m_{3} \exp (\text { int })+m_{4} \exp (- \text { int })\right]\right]
\end{aligned}
$$

$$
N u=\frac{x\left(\frac{\partial T}{\partial z}\right)_{z=0}}{T_{w}-T_{\infty}}=-\frac{k_{n f}}{k_{f}} \operatorname{Re}_{\chi} \theta^{\prime}(0)
$$

Where $\operatorname{Re}_{x}=\frac{U_{r} X}{v_{f}}$ is the Reynolds number.

Thus

$$
\frac{N u}{\operatorname{Re}_{x}}=-\frac{k_{n f}}{k_{f}} \theta^{\prime}(0)
$$

\section{RESULTS AND DISCUSSION}

The numerical calculations are shown in FIG. Described to illustrate the excellent properties of the nanoparticle flow and heat transfer attributes. Table 2-3 and Table 2-3. We have used the information in Table 1 of the thermophysical properties of the base fluid and nanoparticles (copper and alumina) in the numerical calculations. We found the scope of nanoparticle volume fraction $0 \leq \phi \leq 0.2$ range of nanoparticles. The base fluid (water) Prandtl number Pr is held steady at 6,785. We picked $n=10, n t=\pi / 2$ and $\varepsilon=0.001$ while $\phi, \mathrm{R}, \mathrm{S}, \mathrm{K}, \mathrm{M}$,

$Q_{H}$ and $\gamma$ in the present review and are shifted over a distance. Fig. 2 represents the impact of the Hartmann number $\mathrm{M}$ of the velocity dissemination for $\mathrm{Cu}$ - water nanofluid velocity dissemination. It is clear from the calculations that the speed circulation over the boundary layer decreases with an expansion in the Hartmann $\mathrm{M}$ number and decreases near the boundary layer. It reduces the thickness of the boundary layer. As a result, the thickness of the hydrodynamic boundary layer decreases as the magnetic field parameter $\mathrm{M}$ increases for both normal and nanofluid, and therefore the surrounding speed decreases. The explanation for this miracle is that the use of the magnetic field for an electrically conductive fluid offers to rise to a resistance type force called Lorentz force. This pressure appears to impede the movement of the fluid within the boundary layer. The velocity dissemination on the permeable divider is plotted for $\mathrm{Cu}$ water and $\mathrm{Al}_{2} \mathrm{O}_{3}$ in Fig.3 for different estimates of the penetrability parameter $\mathrm{K}$. Clearly the expanded estimations of $\mathrm{K}$ tend to expanding of the velocity on the permeable divider thus upgrade the momentum boundary layer thickness.

The Fig. 4 speaks to the velocity dissemination with the various estimations of Hall parameter $\mathrm{m}$ for $\mathrm{Cu}$-water and $\mathrm{Al}_{2} \mathrm{O}_{3}$. Expanded estimations of $\mathrm{m}$ tend to expanding of the velocity thus improve the momentum boundary layer thickness. The contrary impact is seen with expanding rotation parameter R. Expanding the revolution diminishes the momentum boundary layer thickness (Fig. 5). From Fig. 6 means the velocity profile with the variation from Heat source parameter $\mathrm{Q}_{\mathrm{H}}$. The size of the velocity increments with expanding $\mathrm{Q}_{\mathrm{H}}$ all through the fluid locale. Figs.7 exhibits the impact of the suction/infusion parameter $S$ on the fluid velocity for both nanofluids. The velocity of the fluid over the boundary layer diminishes by expanding suction parameter $\mathrm{S}$ for both nanofluids. Likewise, we find that the velocity is still approaching the corresponding asymptotic motivation for big $\mathrm{z}$ estimates to be $\mathrm{S}$ builds. It merits referencing here that the impact of $S$ on the velocity is increasingly compelling for nanofluids with nanoparticles $\mathrm{Cu}$ and $\mathrm{Al}_{2} \mathrm{O}_{3}$. Thus thickness of the layer diminishes as the suction parameter S. Image. Fig. 8 illustrates the variety of the velocity dispersion for different estimations of $\phi$. From this, the velocity dissemination over the boundary layer diminishes with the expansion of $\phi$. Fig. 9 talks with the different estimates of the convection parameter $\gamma$ for $\mathrm{Cu}-$ water and $\mathrm{Al}_{2} \mathrm{O}_{3}$ - water to the velocity appropriation. Expanded estimations of $\gamma$ tend to expanding of the velocity thus upgrade the energy boundary layer thickness.

Fig.10 exhibits the profiles of temperature for different estimations of $\mathrm{Q}_{\mathrm{H}}$ for copper and alumina. The temperature in the limit layer district diminishes with the expansion in the warmth generation parameter $\mathrm{Q}_{\mathrm{H}}$ and diminishes thickness of Heat layer. These side perspectives fulfill the far field limit conditions asymptotically, which bear the numerical outcomes got. Figure 11 presents the normal profile for temperature diffusion for various estimates of copper and alumina. The figures showed that the temperature in the liquid field decreases as it spreads around the boundary layer and is more extreme outside the plate for both nanoparticles. Consequently, when scaling $\gamma$, the thickness improvements of the thermal boundary layer. In this way we can decipher that the speed of heat transfer decreases with the increase of the convective parameter. This miracle is gradually seen in view of the volume fraction of the nanofluid molecule $\phi$.

These outcomes are in concurrence with the outcomes got by Hamad and Pop[7]. Fig. 12 shows that the variety of suction parameter $\mathrm{S}$ on temperature for both nanofluids. Temperature diminishes with expanding suction parameter S. While expanding $S$ then thickness of heat layer decreases all through the liquid region. The impact of nanoparticle volume fraction parameter $\phi$ on the temperature is appeared in Fig.13 for $\mathrm{Cu}$-water and $\mathrm{Al}_{2} \mathrm{O}_{3}$ - water. The profile of temperature increments with the expansion in in the fraction of nanoparticles denoted by $\phi$. In this way, an increment is found in temperature and reaches to zero far away from the plate layer. 
Table 2 and Table 3 demonstrate separately the differences in skin friction coefficient $\mathrm{C}_{\mathrm{f}}$ and Nusselt number $\mathrm{N}_{\mathrm{u}}$ / Rex with $\mathrm{M}, \mathrm{K}, \mathrm{m}, \mathrm{R}, \gamma, \mathrm{Q}_{H}, S, \phi$. Table 2 shows changes in the skin friction coefficient $\mathrm{C}_{\mathrm{f}}$ and finds that it decreases with expanding parameters $\mathrm{K}, \mathrm{m}$ and $Q_{H}$ increases in the skin friction coefficient with expanding $\mathrm{M}, \mathrm{R}, \mathrm{S}, \gamma, \phi$ for both nanofluids with $\mathrm{Cu}$ and $\mathrm{Al}_{2} \mathrm{O}_{3}$ nanoparticles. In fact, the Nusselt number increases in both nanofluids with the change in all parameters $\gamma, Q_{H}, S, \phi$.

With nanofluids, the variation of the Nusselt number is substantially greater. It should be noted that due to high thermal conductivity compared with $\mathrm{Al}_{2} \mathrm{O}_{3}$, the highest heat transfer value is obtained for $\mathrm{C}_{\mathrm{u}}$. These results are great understanding who are accounted for by Hamad and Pop 7] (Table 4).

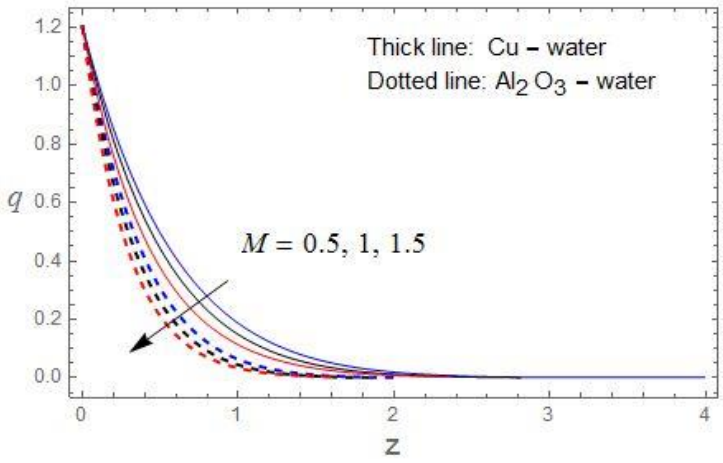

Fig. 2 The velocity frequency with $M$ $K=0.5, m=1, R=0.5, \gamma=2, Q_{H}=1, S=1, \phi=0.05$

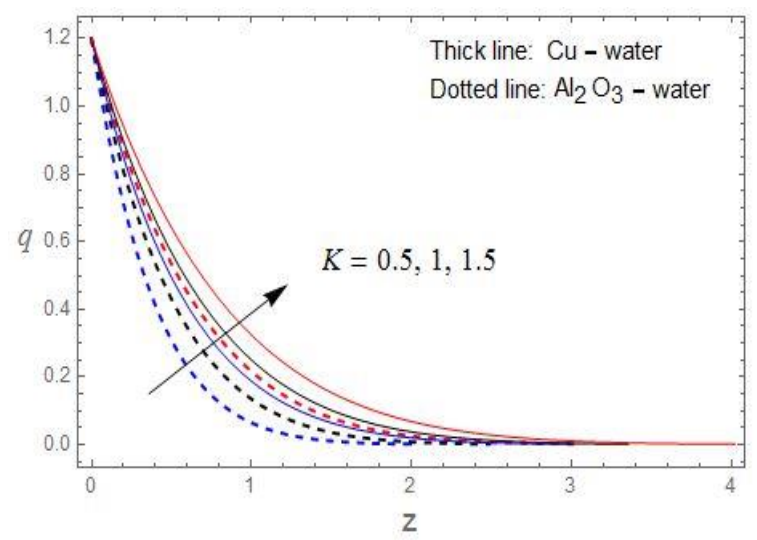

Fig. 3 The velocity frequency with $\boldsymbol{K}$ $M=0.5, m=1, R=0.5, \gamma=2, Q_{H}=1, S=1, \phi=0.05$

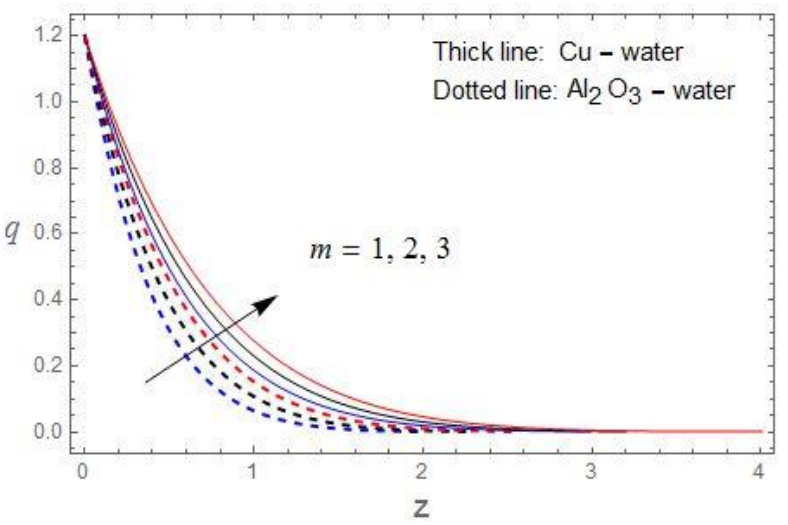

Fig. 4 The velocity frequency with $\boldsymbol{m}$

$M=0.5, K=0.5, R=0.5, \gamma=2, Q_{H}=1, S=1, \phi=0.05$

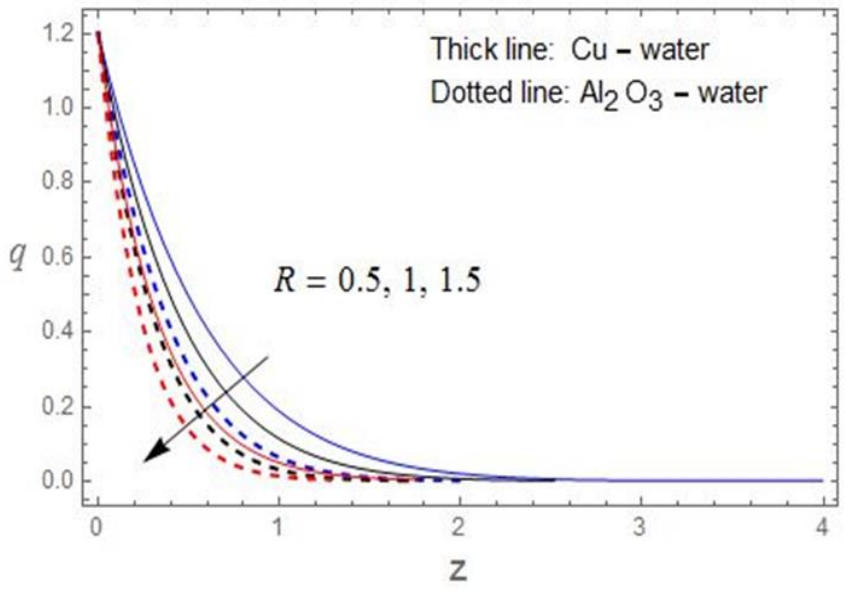

Fig. 5 The velocity frequency with $\boldsymbol{R}$ $M=0.5, K=0.5, m=1, \gamma=2, Q_{H}=1, S=1, \phi=0.05$

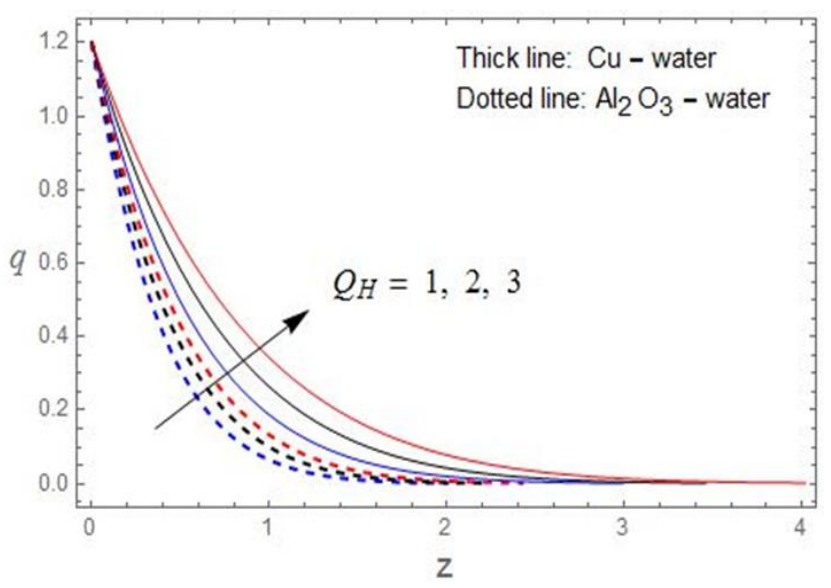

Fig. 6 The velocity frequency with $Q_{H}$ $M=0.5, K=0.5, m=1, R=0.5, \gamma=2, S=1, \phi=0.05$

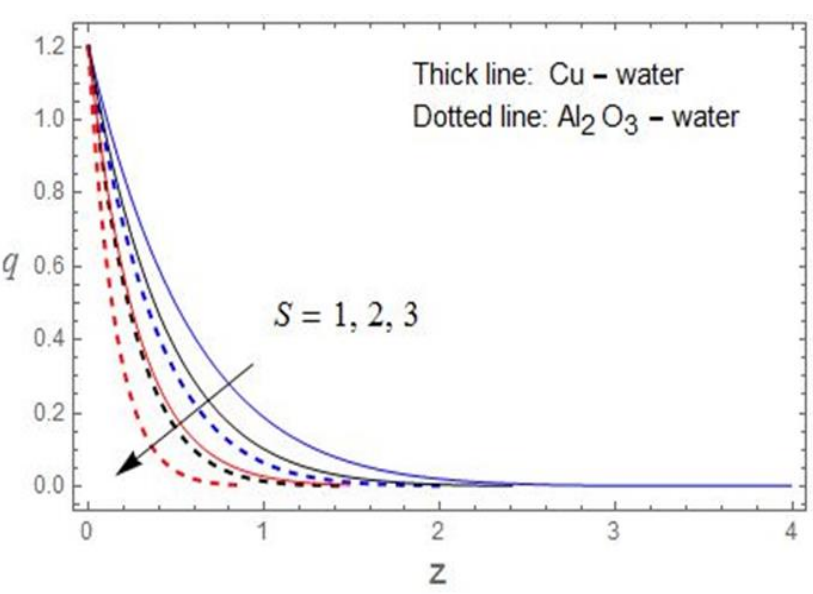

Fig. 7 The velocity frequency with $\boldsymbol{S}$ $M=0.5, K=0.5, m=1, R=0.5, \gamma=2, Q_{H}=1, \phi=0.05$ 
Hall Effects on Unsteady Magneto Hydrodynamic Convection Flow of Nanofluids Past a Rotating Porous Plate

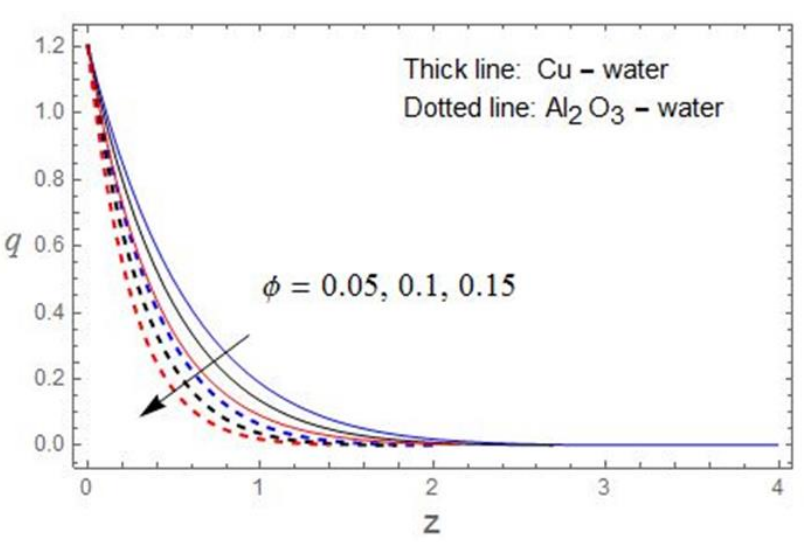

Fig. 8 The velocity frequency with $\phi$ $M=0.5, K=0.5, m=1, R=0.5, \gamma=2, Q_{H}=1, S=1$

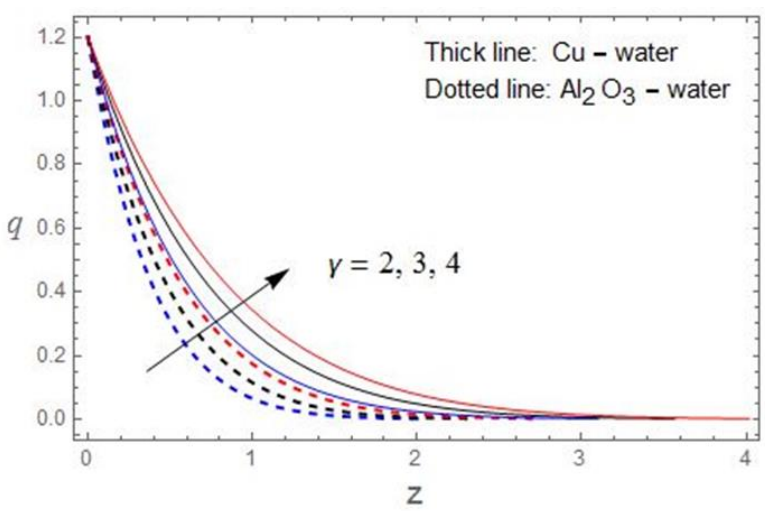

Fig. 9 The velocity frequency with $\gamma$

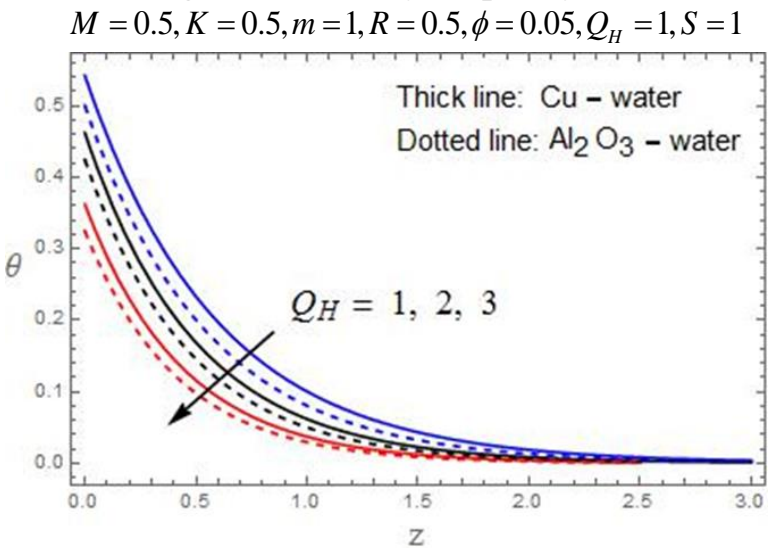

Fig. 10 The temperature frequency with $Q_{H}$

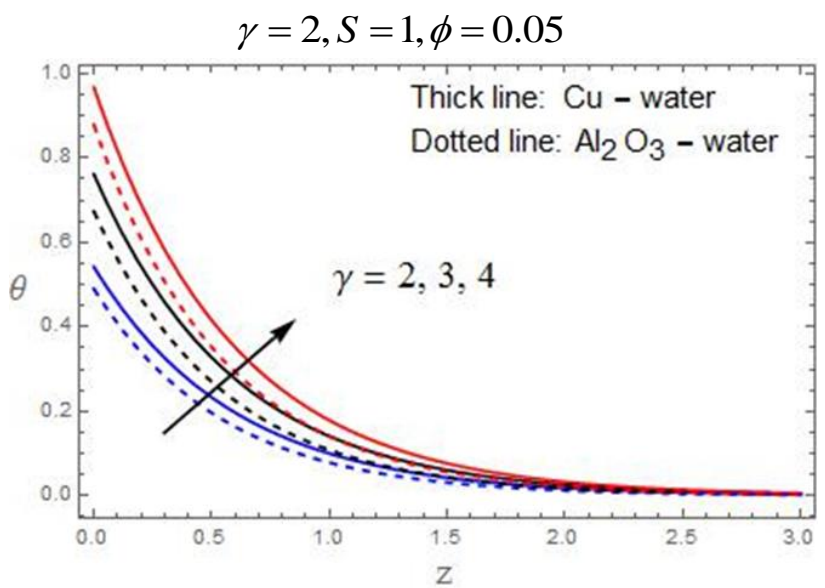

Fig. 11 The temperature frequency with $\gamma$

$$
Q_{H}=1, S=1, \phi=0.05
$$

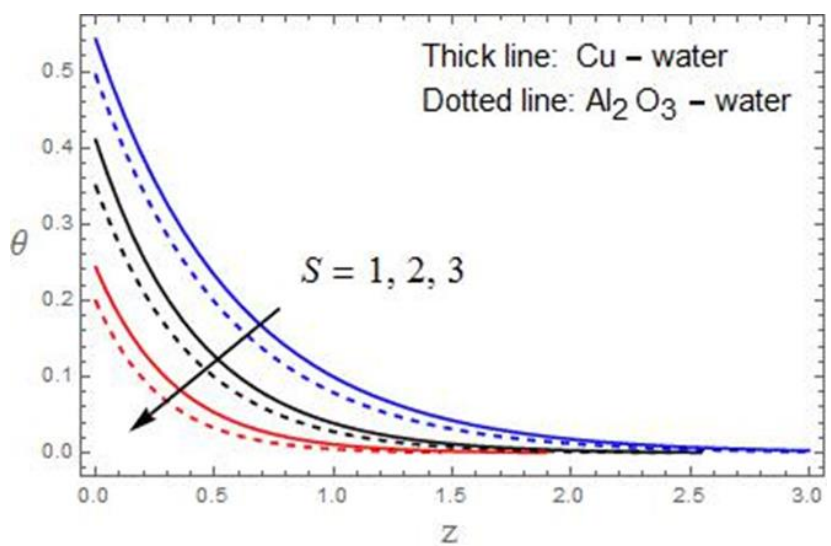

Fig. 12 The temperature frequency with $S$

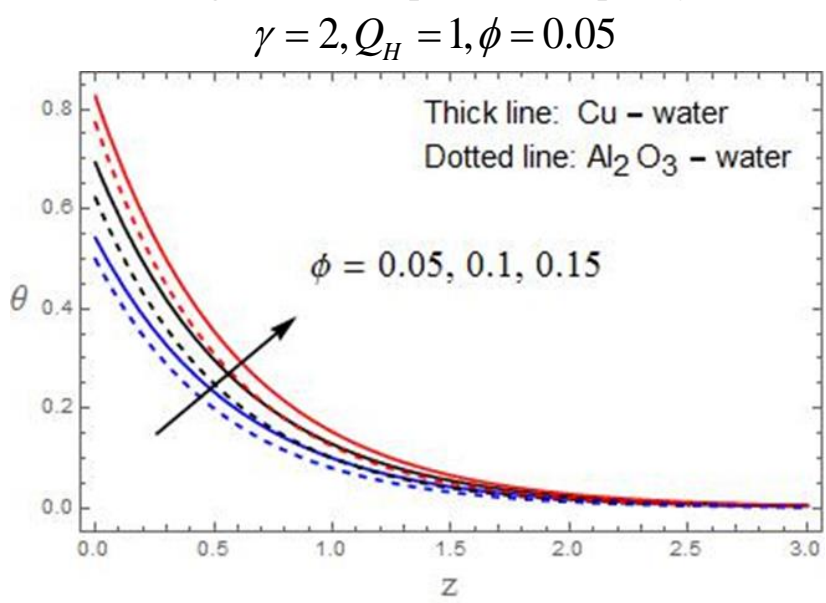

Fig. 13 The temperature frequency with $\phi$

$$
\gamma=2, Q_{H}=1, S=1
$$

Table 1: Standard fluid and nanoparticles have thermo-physical properties

\begin{tabular}{|l|c|c|c|}
\hline $\begin{array}{c}\text { Thermo Physical } \\
\text { properties }\end{array}$ & $\begin{array}{c}\text { Regular fluid } \\
\text { (water) }\end{array}$ & $\mathrm{Cu}$ & $\mathrm{Al}_{2} \mathrm{O}_{3}$ \\
\hline$C_{p}(\mathrm{~J} / \mathrm{kg} \mathrm{K})$ & 4179 & 385 & 765 \\
\hline$\rho\left(\mathrm{kg} / \mathrm{m}^{3}\right)$ & 997.1 & 8933 & 3970 \\
\hline$k(\mathrm{~W} / \mathrm{m} \mathrm{K})$ & 0.613 & 400 & 46 \\
\hline$\alpha \times 10^{7}\left(\mathrm{~m}^{2} / \mathrm{s}\right)$ & 1.47 & 1163.1 & 131.7 \\
\hline$\beta \times 10^{-5}(1 / \mathrm{K})$ & 21 & 1.67 & 0.63 \\
\hline
\end{tabular}

Table 2 Skin friction coefficient

\begin{tabular}{|l|l|l|l|l|l|l|l|l|l|}
\hline$M$ & $K$ & $m$ & $R$ & $\gamma$ & $Q_{H}$ & $S$ & $\phi$ & $\begin{array}{l}C f \\
\mathrm{Cu}- \\
\text { water }\end{array}$ & $\begin{array}{l}C_{f} \\
\mathrm{Al}_{2} \mathrm{O}_{3-} \\
\text { water }\end{array}$ \\
\hline 0.5 & 0.5 & 1 & 0.5 & 2 & 1 & 1 & 0.05 & 2.61072 & 2.45136 \\
\hline 1.0 & & & & & & & & 2.72080 & 2.56425 \\
\hline 1.5 & & & & & & & & 2.89204 & 2.73906 \\
\hline & 1.0 & & & & & & & 2.28173 & 2.11005 \\
\hline & 1.5 & & & & & & & 2.15766 & 1.97899 \\
\hline & & 2 & & & & & & 2.58795 & 2.42794 \\
\hline & & 3 & & & & & & 2.55557 & 2.39520 \\
\hline & & & 1.0 & & & & & 2.67358 & 2.49827 \\
\hline & & & 1.5 & & & & & 2.76488 & 2.56839 \\
\hline & & & & 3 & & & & 2.64518 & 2.48669 \\
\hline & & & & 4 & & & & 2.66783 & 2.50949 \\
\hline & & & & & 2 & & & 2.57982 & 2.41408 \\
\hline & & & & & 3 & & & 2.54005 & 2.38118 \\
\hline & & & & & & 2 & & 3.60562 & 3.21997 \\
\hline & & & & & & 3 & & 4.77669 & 4.13243 \\
\hline & & & & & & & 0.10 & 2.97829 & 2.64416 \\
\hline & & & & & & & 0.15 & 3.37434 & 2.85309 \\
\hline
\end{tabular}


Table 3 Local Nusselt number

\begin{tabular}{|l|l|l|l|l|l|}
\hline$Q_{H}$ & $\gamma$ & $S$ & $\phi$ & $\begin{array}{l}\mathrm{Nu} / \mathrm{Re}_{X} \\
\mathrm{Cu}-\text { water }\end{array}$ & $\begin{array}{l}\mathrm{Nu} / \mathrm{Re}_{X} \\
\mathrm{Al}_{2} \mathrm{O}_{3^{-}} \\
\text {water }\end{array}$ \\
\hline 1 & 2 & 1 & 0.05 & 0.779717 & 0.733261 \\
\hline 2 & & & & 0.844371 & 0.808269 \\
\hline 3 & & & & 0.890440 & 0.860109 \\
\hline & 3 & & & 0.927487 & 0.862487 \\
\hline & 4 & & & 1.024570 & 0.945832 \\
\hline & & 2 & & 0.983947 & 0.919390 \\
\hline & & 3 & & 1.117110 & 1.051350 \\
\hline & & & 0.10 & 0.844161 & 0.787435 \\
\hline & & & 0.15 & 0.900935 & 0.780187 \\
\hline
\end{tabular}

Table 4 Local Nusselt number ( $\left.N u / \mathrm{Re}_{x}\right)$

\begin{tabular}{|l|l|l|l|l|}
\hline$Q_{H}$ & $S$ & $\phi$ & $\begin{array}{l}\mathrm{Cu}-\text { water } \\
\text { Hamad and Pop [24] }\end{array}$ & $\begin{array}{l}\mathrm{Cu}-\text { water } \\
\text { Present results } \\
\gamma \rightarrow 0\end{array}$ \\
\hline 1 & 1 & 0.05 & 0.688574 & 0.733261 \\
\hline 2 & & & 0.744748 & 0.808269 \\
\hline 3 & & & 0.856998 & 0.860109 \\
\hline & 2 & & 0.895578 & 0.919390 \\
\hline & 3 & & 1.002578 & 1.051350 \\
\hline & & 0.10 & 0.785549 & 0.787435 \\
\hline & & 0.15 & 0.884785 & 0.780187 \\
\hline
\end{tabular}

\section{CONCLUSIONS}

In this paper, we consider the impact of the $\mathrm{Cu}$ and $\mathrm{Al}_{2} \mathrm{O}_{3}$ metal nanoparticles on an unstable free MHD convection flow and the hot transfer of the fluid guiding incompressible along a semi-endangered, vertical, penetrable platform that is inserted into the rotating frame of the uniform permeable medium. The ends rising up out of this examination are as per the following:

1. The fluid velocity diminishes with the expansion in Hartmann number, nanoparticle volume tiny proportion, suction and rotation parameter yet impact is invert for Hall and penetrability parameters.

2. An increment in the convective and volume proportion lead to expand the thickness of Heat layer yet inverse impact happens for heat source parameter.

3. The values of $\gamma, Q_{H}, S, \phi$ is increase and to increase the wall temperature gradient for both Copper and Alumina throughout the fluid region.

4. The skin contact coefficient increments with the expansion in the nanoparticle volume part, suction, Hartmann number, rotation parameter and lessens with Hall effects.

\section{REFERENCES}

1. Buongiorno, J., \& Hu, W. (2005, May). Nanofluid coolants for advanced nuclear power plants. In Proceedings of ICAPP (Vol. 5, No 5705, pp. 15-19).

2. Das, K. (2012). Slip flow and convective heat transfer of nanofluids over a permeable stretching surface. Computers \& Fluids, 64, 34-42.

3. Choi, S. U., \& Eastman, J. A. (1995). Enhancing thermal conductivity of fluids with nanoparticles (No. ANL/MSD/CP-84938; CONF-951135-29). Argonne National Lab., IL (United States).

4. Khanafer, K., Vafai, K., \& Lightstone, M. (2003). Buoyancy-driven heat transfer enhancement in a two-dimensional enclosure utilizing nanofluids. International journal of heat and mass transfer, 46(19), 3639-3653.
5. Congedo, P. M., Collura, S., \& Congedo, P. M. (2008, January) Modeling and analysis of natural convection heat transfer in nanofluids. In ASME 2008 Heat Transfer Summer Conference collocated with the Fluids Engineering, Energy Sustainability, and 3rd Energy Nanotechnology Conferences (pp. 569-579). American Society of Mechanical Engineers.

6. Bakr, A. A. (2011). Effects of chemical reaction on MHD free convection and mass transfer flow of a micropolar fluid with oscillatory plate velocity and constant heat source in a rotating frame of reference. Communications in Nonlinear Science and Numerical Simulation, 16(2), 698-710.

7. Hamad, M. A. A., \& Pop, I. (2011). Unsteady MHD free convection flow past a vertical permeable flat plate in a rotating frame of reference with constant heat source in a nanofluid. Heat and mass transfer, 47(12), 1517.

8. Makinde, O. D., \& Aziz, A. (2010). MHD mixed convection from a vertical plate embedded in a porous medium with a convective boundary condition. International Journal of Thermal Sciences, 49(9), 1813-1820.

9. Das, K. (2011). Effect of chemical reaction and thermal radiation on heat and mass transfer flow of MHD micropolar fluid in a rotating frame of reference. International journal of heat and mass transfer, 54(15-16), 3505-3513.

10. Yacob, N. A., Ishak, A., Pop, I., \& Vajravelu, K. (2011). Boundary layer flow past a stretching/shrinking surface beneath an external uniform shear flow with a convective surface boundary condition in a nanofluid. Nanoscale research letters, 6(1), 314.

11. Das, Kalidas. "Flow and heat transfer characteristics of nanofluids in a rotating frame." Alexandria engineering journal 53, no. 3 (2014) 757-766.

12. Krishna, M. V., Swarnalathamma, B. V., \& Chamkha, A. J. (2018). Heat and Mass Transfer on Magnetohydrodynamic Chemically Reacting Flow of a Micropolar Fluid through a Porous Medium with Hall Effects. Special Topics \& Reviews in Porous Media: An International Journal, 9(4).

\section{AUTHORS PROFILE}

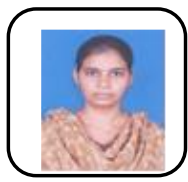

Pushpa Bai Pavar is currently doing Ph.D. in JNTU Anantapuram. She has 3 years research experience and 3 years of teaching experience in UG and PG level.

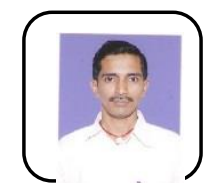

Dr. L. Hari Krishna acquired Ph.D degree in the area of "Fluid Dynamics" from JNTU, Anantapur in 2010. He obtained his M.Phil from M.K.University, Madhurai in the year 2004. He obtained his M.SC degree in mathematics from S.V.University, Tirupati in the year 1998. He has 19 years of experience in teaching in UG and PG and 9 years of research experience.

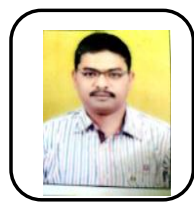

M. Suryanarayana Reddy is working as Assistan Professor in Department of Mathematics, JNTUA College of Engineering, Pulivendula. He obtain Ph.D. from JNTU Anantapuram. He has 19 years of experience in teaching. 